\title{
On the Unified Description of the Motion of Physical Bodies
}

\author{
Khachatur Kirakosyan \\ Institute of Chemical Physics, Armenian National Academy of Sciences, Armenia \\ Email: khkirakosyan@gmail.com
}

\begin{abstract}
It is supposed that beginning with the definite hierarchical level the physical bodies possess the same structural element, which specifies the same mechanism of motion and the same quantitative apparat of describing the motion. From the proposed mechanism, the minimal intervals of space and time necessary for the quantitative description of motion are defined and the structural content of the "mass" notion and the parameters related to it revealed. The unified equation of motion is proposed.
\end{abstract}

Keywords: Structure, space, time, motion, mass, dualism, invariance.

\section{Introduction}

All the Physics is to be considered in the light of the science of motion: of macroworld - the Classical Mechanics (CM), the microworld and world of high velocities, the Quantum Mechanics (QM) and the Special Theory of Relativity (STR.) Notions and laws lay in the basis of the quantitative description phenomena of any field of natural science. Nevertheless, there is no basis today that unites the conceptual directions of the descriptions of the motion $[1,2,3,4]$ the content of the basic concept of the dynamics of mass is not revealed $[5,6,7,8]$ there are some uncertainties related to the use of vanishing intervals of space $(\Delta l \rightarrow 0)$, time $(\Delta t \rightarrow 0)$ and sizes of material point frequently considered as an object of motion. From the physical point of view, the transfer to the limits $\Delta l \rightarrow 0, \Delta t \rightarrow 0$ implies the penetration deep into the structure of matter; besides, the material point under consideration is transformed into the microparticle with the complex structure. Consequently, with the aim in view to reveal the content and the calculation of mass there is a need to establish relation between the regularities of motion and the structure of microparticles.It is assumed that the solution of this and a great number of similar problems is associated with the penetration deep into the structure of matter by a rank below the known hierarchical level. The history of Physics is the history of the discovery of the fundamental properties of matter, the history of penetration into the expanses of the Universe, and into the deep levels of the structure of matter. In the approach of $[9,10]$, which is called the Structural Theory (ST), and is adequate to the deeper hierarchical levels of the structure of matter than the generally accepted ones, the content of the fundamental properties of matter and the laws relating them are reduced to the clearly predicted internal material relations. Regarding the science of motion, the goal of the ST is to uncover the mechanism of motion of physical bodies, to establish a relation between the generally accepted concepts and the laws of mechanics and the parameters characterizing the mechanism of motion.

In ST it is assumed that starting from some hierarchical level, all the physical bodies have the same structure element related to the mechanical motion and it is shown that the obtained equations of motion are the unifying base for initial equations of the Mechanics, Electrodynamics, and Thermodynamics. Below, based on the model of mentioned structure element, the unified quantitative principle is obtained of moving bodies description with the well-grounded choice of the minimal intervals of space and time, and the structural content of "mass" notion and parameters related to it is revealed, is provided the conditions of transition from the ST-equations to the equations of the CM, QM, and STR.

In the ST which is not operating with the idea of physical vacuum, any processes with the participation of microworld particles, by the analogy with the chemical transformations, are reduced to the connection, decay or exchange by the composite elements. From the beginning, the hypothesis is put forward on the existence of some particles of conventionally minimum hierarchical level ( $\varepsilon$-particles), the attribute of which is their ability to interact in pairs with each other. It is accepted that the elementary act of interaction between the $\varepsilon$-particles ( $\varepsilon$-act) takes part with the strictly defined duration on the strictly 
defined distance; in addition, because of the $\varepsilon$-act the particles pass same distance, creating new pairs. Hence, by counting the sequentially realized number of $\varepsilon$-acts one can determine both path and time. By operating with the dimensional parameters, the $\varepsilon$-intervals of length and time are taken as $\xi_{d} \mathrm{~cm}$ and $\xi_{t}$ s. Three types of $\Delta$-elements are modelled with the use of $\varepsilon$-particles: $\Delta_{i}, \Delta_{j}$ and $\Delta_{k}$ oscillating along three mutually perpendicular directions. Here and below, the indexes " $i$ ", " $j$ " and " $k$ " denote the directions of motion. In each $\Delta$-element, the content of $\varepsilon$-pairs and they mutual ordering are chosen in such a way, that $\Delta_{i}, \Delta_{j}$ and $\Delta_{k}$ elements are mutually recognizable in the bound state. With the $\Delta$-elements, the $\Delta$-pairs are modelled both with the same $\left(2 \Delta_{i}, 2 \Delta_{j}, 2 \Delta_{k}\right)$ and the different directions of oscillations. The pair of identical $\Delta$-elements is characterized by the $\alpha_{0}$-fold repetition of oscillations with an amplitude $H_{c}$, that is, by the total number of $\varepsilon$-acts

$$
H_{00}=\alpha_{0} \cdot H_{c}
$$

where

$$
\alpha_{0}=\sum_{n=1}^{7} n^{2}=\sum_{n=1}^{7} \sum_{l=0}^{n-1}(2 l+1)=140
$$

is the number of subsequentially realized states of $\Delta$-pairs, $n$ and $l$ are analogues of the principal and azimuthal quantum numbers for the given hierarchical level.

From three $\Delta$-pairs, the $\gamma$-particles of various destination are modelled, which are the base of the known particles of micro- and macroworld. To demonstrate this, let us cite as an example the models of electron $\left(e^{-}\right)$, positron $\left(e^{+}\right)$and the mechanism of interaction between them. The electron and positron are modelled by each of the two $\gamma$-particles: $\gamma_{e 0}$ and $\gamma_{p 0^{-}}$are the bases and $\gamma_{e E}$ and $\gamma_{p E}$ are particles of the $\Delta$-content

$$
\gamma_{e 0}=2 \bar{i} 2 \bar{k}(\bar{i} \bar{k}), \gamma_{e E}=4 j(\bar{i} k) ; \gamma_{p 0}=2 i 2 \bar{k}(i \bar{k}), \gamma_{p E}=4 \bar{j}(i k)
$$

where the symbols of $\Delta_{i}, \Delta_{j}$ and $\Delta_{k}$-particles are replaced here and below by their corresponding indices, the vinculum over the symbol of $\Delta$-elements denotes that they are moving in the backward direction, the indices " $e$ " and " $p$ " denote that the $\gamma$ - particle belongs to $e^{-}$or $e^{+}$.

Doing the shuttle motion relative to bases, the $\gamma_{e E}$ - and $\gamma_{p E^{-}}$particles interact periodically with them according to the scheme

$$
\begin{aligned}
& 4 j(\bar{i} k)+2 \bar{i} 2 \bar{k}(\bar{i} \bar{k}) \rightleftarrows 2 j 2 \bar{i} 2 \bar{k}+2 j 2 \bar{i} 2 \bar{k} \\
& 4 \bar{j}(i k)+2 i 2 \bar{k}(i \bar{k}) \rightleftarrows 2 \bar{j} 2 i 2 \bar{k}+2 \bar{j} 2 i 2 \bar{k}
\end{aligned}
$$

where the symbol $\rightleftarrows$ indicates that the interaction is reversible; with the participation of $\gamma_{E}$ particles, the electrostatic interaction between slow $e^{-}$and $e^{+}$is also realized, which is stressed by the index " $E$ " near $\gamma$. The initial stage of electrostatic interaction between slow $e^{-}$and $e^{+}$is reduced to the exchange $2 j$ and $(i k)$ pairs between $\gamma_{E^{-}}$particles and $\gamma_{0^{-}}$bases of partners according to scheme

$$
\begin{aligned}
\frac{2 \bar{i} 2 \bar{k}(\bar{i} \bar{k})}{4 \bar{j}(i k)} & \longleftrightarrow \frac{2 j_{i} 2 \bar{i} 2 \bar{k}}{2 \bar{j}_{i} 2 i 2 k} \\
\frac{4 j(\bar{i} k)}{2 i 2 \bar{k}(i \bar{k})} & \longleftrightarrow \frac{2 j 2 \bar{i} 2 k}{2 \bar{j}_{i} 2 i 2 \bar{k}}
\end{aligned}
$$

where the $\gamma$-particles of $e^{-}$are given in the numerator, and $\gamma$-particles of $e^{+}$are given in the denominator. The indices " $i$ " near symbol " $j$ " denote that the $2 j$ - pairs are introduced in the content of $\gamma$ - particles as a result of interaction with the exterior partners. For $\Delta$-elements, the change of nominator and denominator results in the change of direction of motion to the opposite. In the of interaction, the bases of $e^{-}$and $e^{+}$are characterized by $\Delta$-content: $\gamma_{e i}=2 j_{i} 2 \bar{i} 2 \bar{k}, \gamma_{p i}=2 \overline{j_{i}} 2 i 2 \bar{k}$, derivatives on $\gamma_{E}$-particles: $\gamma_{e M}=2 j 2 \bar{i} 2 k$ and $\gamma_{p M}=2 \bar{j} 2 i 2 k$.

For further approach, the positronium is formed from the right sides of (5), which later decays into the two photons with the opposite directions of motion:

$$
\left[\frac{2 j 2 \bar{i} 2 \bar{k}, 2 j 2 \bar{i} 2 k}{2 \bar{j} 2 i 2 k, 2 \bar{j} 2 i 2 k}\right]=\frac{2 j 2 \bar{i} 2 \bar{k}}{2 \bar{j} 2 i 2 \bar{k}}+\frac{2 j 2 \bar{i} 2 k}{2 \bar{j} 2 i 2 k}
$$


where the direction of motion of a specific photon is defined by the presence in the nominator (the world of $e^{-}$) and in the denominator (the world of $\left.e^{+}\right)$of the $\Delta$-pairs with the same direction of motion $(2 \bar{k}$ and $2 k$ for given variant).

The general content of $2 j 2 i 2 k$ obtained at the self-interaction (4), electrostatic interaction (5) and annihilation (6) are called the $\gamma_{0 i^{-}}$particles; it is assumed that the presence of these $\gamma_{0 i^{-}}$particles in all physical bodies results in the generality of quantitative regularities of their motion.

The bound state in $\gamma$-particles is caused by the self-consistent interaction and motion: the $\varepsilon$-particles in $\Delta$-elements, the $\Delta$-elements in the $\Delta$-pairs, and $\Delta$-elements themselves in the $\gamma$-formations. To provide the stability at the modelling of $\gamma_{o i}$-particles, the oscillations of $2 i$ - and $2 k$-pairs are chosen with the phase difference equal to $\pi / 2$; because of this they are called the self-oscillations. At certain conditions, the $2 k$ - and $2 j$-pairs switch the roles. In the case of self-interaction, the $2 j$-pair is selected relative to the oscillations of $2 i$-pair with the phase $3 \pi / 2$, which also results in the closed trajectories; thereby, the base of the particle is always in the restricted part of the space.

The presence of the phase $\pi / 2$ results in the formation of closed curvilinear trajectories as a result of which it is assumed that the computed amplitudes of oscillations $H_{c}(1)$ are changing in $\chi_{c}$ times, that is, the $\Delta$-pairs in the content of $\gamma_{0 i}$-particles are characterized by the total number of $\varepsilon$-acts

$$
H_{0}=\alpha_{0} H_{c} / \chi_{c}=\alpha_{c} H_{c},
$$

where is denoted

$$
\alpha_{c}=\alpha_{0} / \chi_{c}
$$

\section{The Main Equation of Motion}

When describing the regularities of motion, the $\gamma_{0 i}$-particles themselves can be considered as an intrinsic frame of reference (IFR) using the orthogonally related directions of oscillations of $\Delta$-pairs as the axes of reference, as for the time, it can be defined by calculating the sequentially realized $\varepsilon$-acts along the all directions of interaction. The numerical values of coordinates for given time are defined by the number of sequentially realized $\varepsilon$-acts along the corresponding direction.

The motion of $\gamma$-particles of general content $2 j 2 i 2 k$ is characterized by sequentially realized states

$$
2 i[2 j 2 k] \rightarrow 2 \bar{k}[2 i 2 \bar{j}] \rightarrow 2 \bar{i}[2 \bar{k} 2 j] \rightarrow 2 k[2 \bar{i} 2 \bar{j}] .
$$

We now turn to the quantitative description of the mechanism of motion. According to the given contents, the initial stage of displacement is caused by the leading $2 i$-pair with the netting of perpendicular surface oriented along the direction of displacement, taken in the square brackets $2 j 2 k$-quartet. In what follows, the displacement is realized by the $2 \bar{k}, 2 \bar{i}$ and $2 k$-pairs with the netting of the orthogonal surfaces oriented along the direction of propagation by $2 i 2 \bar{j}-, 2 \bar{k} 2 j-$ and $2 \bar{i}, 2 \bar{j}-$ quartets. It follows from the mechanism that some temporal volume $V_{t}$, which is called the trajectorial volume, is formed from the traces of trajectories in the forward direction. The total number of spent $\varepsilon$-acts per formation of volume per total cycles of displacement (9) with allowance of formula (7) can be defined by the mixed product of vectors

$$
2 H_{i 0}\left[H_{j 0} \times H_{k 0}\right]+2 H_{k 0}\left[H_{i 0} \times H_{j 0}\right]=H_{j 0}\left[2 H_{k 0} \times 2 H_{i 0}\right]
$$

where $H_{i 0}, H_{j 0}$ and $H_{k 0}$ are the $\alpha$-fold repetition of amplitude of oscillations of $2 i, 2 j$ and $2 k-$ pairs. The product of vectors is taken in the square brackets with the symbol of multiplication " $\times$ ", the number of $\varepsilon$-acts spent for the formation of the same volume $V_{t}$ is defined as a result of displacement of the leading $2 j$-pair with the netting of the orthogonal surface by the $2 k, 2 i-$ quartet. The elementary volume $d V_{t}$ derived because of the circulation of oriented surface $S$ over the elementary path $d l$ will be given by the equation

$$
d V_{t}=S d l=H_{i s}^{2} d l
$$

where

$$
S=S_{i}+S_{k}=H_{i s}^{2}, S_{i}=H_{j i} \times H_{k i}, S_{k}=H_{i i} \times H_{j i},
$$

$d l$ is a sum of elementary paths from the leading $2 i-, 2 k$-pairs 


$$
d l=d l_{i}+d l_{k}
$$

with the additional index " $S$ " here and below is stressed that the given quantity is an oriented surface. Summing up the elementary volumes (11), let us define the circulation $C_{S}$ of axial vector $S$ (12) over the closed path $L$; that is $C_{S}=\sum S d l=\oint_{L} S d l$. From the definition of vector

$$
\operatorname{curl} S=\lim _{S_{j} \rightarrow 0} \frac{\oint_{L} S d l}{S_{j}}
$$

the volume $V_{t}$ will be calculated with the use of integrals

$$
\oint_{L} S d l=\oint_{S} \operatorname{curl} S d S_{j}
$$

where $S_{j}$ is an orthogonal surface netted by the $2 i 2 k$-quartet.

Consider $2 j$-pair as a leading one. The elementary volume $d V_{t}$ with the allowance of the right part of equality (10) will be defined as

$$
d V_{t}=l_{j c} d S_{j}
$$

where $l_{j c}=H_{j c} \xi_{d}$. Hence, the volume $V_{t}$ is defined by the integral

$$
V_{t}=\oint_{S_{j}} l_{j c} d S_{j}
$$

Comparing the equations (15) and (17), one can conclude that

$$
\operatorname{curl} S=l_{j c}=H_{j c} \xi_{d}
$$

According to the mechanism of motion considered above, the centers of orthogonal surfaces netted by the $2 j 2 k$ - and $2 i 2 j-$ quartets are displaced over the circle formed by the $2 i-$ and $2 j-$ pairs, respectively, using the equation of oscillations of $2 i-, 2 j$-and $2 k-$ pairs; $r_{i t}=r_{i c} \cos \omega t, r_{k t}=r_{k c} \sin \omega t, r_{j t}=$ $r_{j c} \sin \omega t$ are the concluding trajectories of motion of particles are given by the parametric equations

$$
\begin{aligned}
& l_{i t}=\left(r_{i c}+r_{j c} \cos \omega_{j} t\right) \cos \omega t \\
& l_{k t}=\left(r_{k c}+r_{j c} \cos \omega_{j} t\right) \sin \omega t \\
& l_{j t}=r_{j c} \sin \omega_{j} t
\end{aligned}
$$

where $r_{c}=H_{c} \xi_{d} / 2, \omega=2 \pi / T$, the period of oscillation $T$ is the same for all the $\Delta$-pairs because of the equality of moduli $\left|H_{i c}\right|=\left|H_{k c}\right|=\left|H_{j c}\right|, t$ is time, $\xi_{d}$ is the dimensional value of the $\varepsilon$-interval of length.

Starting from equations $(7)$ and (19), the quantity $L$ can be defined only by the oscillations of $2 i$ and $2 k-$ pairs:

$$
L=\alpha_{c} 2 \pi r_{c},
$$

And the quantity $S_{j}$ will be defined by the curvilinear integral

$$
S_{j}=\frac{1}{2} \int_{0}^{T}\left(l_{k t} d l_{i t}-l_{i t} d l_{k t}\right)=\pi H_{0 S}^{2} \xi_{d}^{2}=\pi \alpha_{c}^{2} H_{c S}^{2} \xi_{d}^{2}
$$

where $H_{c S}^{2}=H_{k c} \times H_{i c}$.

Using quantities $L(20)$ and $S_{j}$ as the limits of integration of equation (15), the quantity $V_{t}$ for $j_{0 i}$-particles obtained by self-interaction will be given by formulas

$$
\alpha_{c} 2 \pi r_{c}\left[\alpha_{c}^{2} H_{c s}^{2}\right]=\alpha_{c} H_{j c}\left[\pi \alpha_{c}^{2} H_{c S}^{2}\right]=H_{0} \pi H_{0 S}^{2}
$$

In the case of electrostatic interaction (5), some number of $\varepsilon$-acts is spent for the removal of $j_{E}$-particles from their bases, because of which the $2 j_{i}$ pairs, introduced into the content of $j_{0 i}$-particles, have $\chi_{i}$-times smaller amplitude, that is,

$$
H_{j i}=H_{j c} / \chi_{i}
$$


or, taking into account formula (7), for general case

$$
\alpha_{c} \chi_{i} H_{i}=H_{0}
$$

Dividing and multiplying the left-hand side of equation (22) by $\alpha_{c}^{2} \chi_{i}^{2}$, we obtain

$$
\alpha_{c} 2 \pi \chi_{i}^{2} \alpha_{c}^{2} r_{c}\left[H_{i S}^{2}\right]=\alpha_{c} H_{j c} \pi H_{0 S}^{2},
$$

from which it follows that at the small values of $H_{j i}$ the trajectorial volume is formed by the circulation of the surface

$$
H_{i S}^{2}=H_{i i} \times H_{j i}+H_{j i} \times H_{k i}
$$

along the circle of length

$$
2 \pi r=2 \pi \chi_{i}^{2} \alpha_{c}^{2} r_{c}
$$

where

$$
r=\chi_{i}^{2} \alpha_{c}^{2} r_{c}
$$

Dividing both sides of the equation $(25)$ by $\chi_{i}$, let us define the value of trajectorial volume caused by the potential of an extraneous interaction

$$
2 \pi \chi_{i} \alpha_{c}^{2} r_{c} H_{i S}^{2}=\lambda H_{i S}^{2}=H_{i j} \pi H_{0 S}^{2}
$$

where the path of circulation $\lambda$ is defined by the formula

$$
\lambda=2 \pi \chi_{i} \alpha_{c}^{2} r_{c}
$$

Taking into the account the formula (23) and summing (25) and (29), let us define the trajectorial volume caused by the self and extraneous interactions

$$
(\alpha 2 \pi r+\lambda) H_{i S}^{2}=\left(\chi_{i} \alpha_{c}+1\right) H_{j i} \pi H_{0 S}^{2}
$$

The interpretation of equation (15) differs from the interpretation of the analogous Stocks equation by the fact that in this case the motion of single particle is described, and the volume formed from the traces of single $j_{0 i}$ is calculated, and not the statistical system or medium with many particles.

The algebraic specification (15), (22), (25), (29) and (31) is called the basic equation of motion. According to these equations, the motion of physical bodies is the sum of transversal component with the curvilinear closed trajectory (the circulation path) with the orthogonal surface $H_{i S}^{2}$ and the transversal component with the orthogonal surface $\pi H_{0 S}^{2}$ (the right hand sides of equation of motion). So, it follows from the equation (29) that the passing of longitudinal path with the perpendicular surface $\pi H_{0 S}^{2}$ corresponds to the transversal path $\lambda$ with the orthogonal surface $H_{i S}^{2}$.

Overall, the numerical value of $\operatorname{curlH}_{i S}^{2}$ the behavior of the particle is defined by the limits of integration of equation (15). 2j-pair is changed by such sequence, the third equation in the system (19) is given by the equality $l_{j}=V_{j} t$ ( $V$ is the velocity of particle, $t$ is time), the particle stops to be in the limited part of space and moves in space along the direction of curl $_{i s}^{2}$. The resulting averaged direction of motion of the many particle system is defined by the sum of projections curl $_{i S}^{2}$ from each of the particle separately.

\section{Inherent Kinematic Characteristics and the Velocity of Motion of Physical Bodies}

In equations (29), (22) and (31), by passing of least longitudinal paths the results of interaction are revealed:

$$
l_{i}=H_{i} \xi_{d}, l_{0}=H_{0} \xi_{d}, l_{0 i}=\left(H_{0}+H_{i}\right) \xi_{d},
$$

to which correspond the least transversal paths defined by equations

$$
\lambda=\frac{H_{i} \pi H_{0}^{2} \xi_{d}}{H_{i}^{2}}=\frac{\pi H_{0}^{2} \xi_{d}}{H_{i}}, \lambda_{0}=\frac{\pi H_{0}^{2} \xi_{d}}{H_{0}}=\pi H_{0} \xi_{d}, \lambda_{0 i}=\frac{\left(H_{0}+H_{i}\right) \pi H_{0}^{2} \xi_{d}}{H_{i S}^{2}} .
$$


As a generalizing parameter of longitudinal and transversal motions, the path $\lambda_{\Sigma}$ is introduced in form of

$$
\lambda_{\Sigma}=\frac{\left(H_{0}+H_{i}\right) \pi H_{0 S}^{2} \xi_{d}}{H_{0}^{2}+H_{i}^{2}}=\frac{\left(H_{0}+H_{i}\right) \pi H_{0 S}^{2} \xi_{d}}{\left(H_{0}+H_{i}\right)^{2}}=\frac{\pi H_{0 S}^{2} \xi_{d}}{H_{0}+H_{i}}
$$

where, with the allowance of the condition $H_{i} \perp H_{0}$, it is assumed that $H_{i} \cdot H_{0}=0$.

Because the longitudinal component of motion is realized by the "netting" of perpendicular surface $\pi H_{0 S}^{2}$, the paths $(32)$ are overcome at the time intervals

$$
\tau_{i}=H_{i} \xi_{\tau}, \tau_{0}=H_{0} \xi_{\tau}, \tau_{0 i}=\left(H_{0}+H_{i}\right) \xi_{\tau}
$$

where the new coefficient of time dimension is introduced

$$
\xi_{\tau}=\pi H_{0}^{2} \xi_{t}
$$

It is not difficult to see in (35) that the dimensionless parts of time represent the corresponding trajectorial volume. Because both sides in equations (29), (22) and (31) define the same volume, the corresponding time of paths passing in (32) and (33) also should be equal.

Taking into account that the trajectory of displacement caused by the oscillations of intrinsic $\Delta$-pairs (19) is always closed, the total displacement of $\gamma_{0 i}$-particles will be caused only by the exterior interaction and there by the velocity of longitudinal motion is represented by the relationship of path $l_{i}$ and time $t_{0 i}$ :

$$
v=\frac{H_{i} \xi_{d}}{\left(H_{0}+H_{i}\right) \xi_{\tau}}=\frac{H_{i}}{H_{0}+H_{i}} c
$$

where $c$ is denoted as

$$
c=\xi_{d} / \xi_{\tau}
$$

Because of its $\Delta$-content (6) the longitudinal motion of photon is realized only by one direction with the orthogonal surface $H_{0 S}^{2}$, thereby the path $H_{i} \xi_{d}(32)$ is overcome in time $\tau_{i}(35)$, correspondingly, the velocity of motion of photon will be given by the relation $H_{i} \xi_{d} / H_{i} \xi_{\tau}=\xi_{d} / \xi_{\tau}=c$, that is, by the formula (38). Thus, the velocity of photons is always constant because of the constant $\xi_{d}$ and $\xi_{\tau}$. Multiplying the dimensionless components of transversal paths (33), for time, we obtain one more series that is characteristic:

$$
\tau_{i}=\frac{H_{i} \pi H_{0}^{2} \xi_{\tau}}{H_{i}^{2}}=\frac{\pi H_{0}^{2} \xi_{\tau}}{H_{i}} ; \tau_{0}=\pi H_{0} \xi_{\tau} ; \tau_{0 i}=\frac{\left(H_{0}+H_{i}\right) \pi H_{0}^{2} \xi_{\tau}}{H_{i}^{2}}
$$

It is obvious that with the use of the reciprocal quantities of temporal intervals (39), the frequencies of manifestation of paths (33) can be determined:

$$
\nu_{i}=\frac{H_{i} \xi_{\nu}}{\pi H_{0}^{2}} ; \quad \nu_{o}=\frac{\xi_{\nu}}{\pi H_{0}} ; \quad \nu_{0 i}=\frac{H_{i}^{2} \xi_{\nu}}{\left(H_{0}+H_{i}\right) \pi H_{0}^{2}}
$$

where the coefficient with the dimension frequency is denoted by $\xi_{\nu}$

$$
\xi_{\nu}=\xi_{\tau}^{-1} .
$$

It follows from the definitions (33), (34), and (37) that the quantities $\lambda$ and $\lambda_{\Sigma}$ are related by the equation

$$
v \lambda=c \lambda_{\Sigma}
$$

The parameters with the variable $H_{i}$, given in formulas $(32),(33),(34),(39)$ and (40) and being related with the structure and mechanism of motion of $\gamma_{0 i}$-particles are the basis of kinematics because of which they are called the proper kinematical characteristics of physical bodies.

Dependent on hierarchical level of problems considered, the duration of processes can be estimated by the least intervals of time $\Delta t$, equal to $\xi_{t}, \xi_{\tau}$; the quantities from the series (35) and (39), the least intervals of the space are chosen correspondingly, because the time and length in this case are measured by the unified instrument. In general, the axes of $l=f(t)$ dependences are constructed from the definite numbers of given least intervals; thereby the axes of space and time became exclusively numerical. 
For $\gamma_{0 i}$-particles, as an integral structural unit, the least intervals of path and time $l_{i}$ and $t_{0 i}$ are characteristic, that is, the operation with the least intervals $l_{i}$ and $t_{0 i}$ is equivalent to ascribing the role of material point to the $\gamma_{0 i}$-particles. By contact of these points, the numerical axes of the dependence $l=f(t)$ are constructed. In this system of coordinates, any interval of path and time can be represented as $n$-fold repetition of $l_{i}$ and $t_{0 i}$, that is, $\Delta l=\Delta n l_{i}=\Delta n H_{i} \xi_{d}, \quad \Delta t=\Delta n \tau_{0 i}=\Delta n\left(H_{0}+H_{i}\right) \xi_{\tau}$. Hence, the conventional definition of velocity $v=\lim _{\Delta t \rightarrow 0} \frac{\Delta l}{\Delta t}$ will be changed by $v=\lim _{\Delta t \rightarrow \tau_{0 i}} \frac{\Delta l}{\Delta t}$, that is, we get rid of the uncertainty contained in the conditions. Simultaneously, the uncertainty contained in the conditions vanishes $\Delta l \rightarrow 0$, because in the approach set forth, the unified tool defines the time and path - by the computation of the number of $\varepsilon$-acts along all the directions of motion.

It emerges from the foregoing that the periodic repetition of quantity $H_{i}$ is a criterion of the uniform motion of physical bodies with the constant velocity. Denoting the path as $l=n l_{i}$ and time as $t=n \tau_{0 i}$ and with the allowance of series (32)-(35), the velocity of longitudinal motion will be defined by the equation

$$
v=\frac{d l}{d t}=\frac{H_{i} \xi_{d} d n}{\left(H_{0}+H_{i}\right) \xi_{\tau} d n}=\frac{H_{i}}{H_{0}+H_{i}} c
$$

that is, analogous to the equation (37). The $n$-fold repetition of $H_{i}$ is the $n$-fold manifestation of the integrity of particle, thereby replacing in equations (8), (23) and (24) $\chi_{i}$ by $n$, we will define how large is the integrity of $\Delta$-pairs of given type along the direction of interaction.

$$
n H_{i}=H_{c}, n \alpha_{o} H_{c} / \chi_{c}=n \alpha_{c} H_{i}=H_{0}
$$

Using these formulas, the equation of motion (25), (29) and (31) can be written as

$$
\begin{gathered}
\alpha_{c} 2 \pi n^{2} \alpha_{c}^{2} r_{c} H_{i S}^{2}=\alpha_{c} H_{j c} \pi H_{0 S}^{2} \\
2 \pi n^{2} \alpha_{c}^{2} r_{c} H_{c} H_{i S}^{2}=H_{j i} \pi \alpha_{c} H_{0 S}^{2} \\
(\alpha 2 \pi r+\lambda) H_{i S}^{2}=\left(n \alpha_{c}+1\right) H_{j i} \pi H_{0 S}^{2}
\end{gathered}
$$

Writing the dependence of formulas (27) and (30) on $n$.

$$
\begin{gathered}
2 \pi r=2 \pi n^{2} \alpha_{c}^{2} r_{c} \\
\lambda=2 \pi n \alpha_{c}^{2} r_{c}
\end{gathered}
$$

we obtain

$$
n \lambda=2 \pi r
$$

correspondingly, the equation (47) can be represented in the form

$$
\left(n \alpha_{c}+1\right) \lambda H_{i S}^{2}=\left(n \alpha_{c}+1\right) H_{j i} \pi H_{0 S}^{2}
$$

or

$$
\lambda H_{i S}^{2}=H_{j i} \pi H_{0 S}^{2}
$$

Using formulas (37), (38) and (49) the velocity of longitudinal $(v)$ and transversal $\left(v_{n}\right)$ motion can be written as

$$
v=\frac{c}{n \alpha_{c}+1}, \quad v_{n}=\frac{n^{2} \alpha^{2}}{n \alpha_{c}+1} c
$$

from which at $n \alpha \gg 1$, which is equivalent to the condition $H_{i} \ll H_{o}$ we obtain

$$
v=\frac{c}{n \alpha_{c}}, \quad v_{n}=\frac{n^{2} \alpha_{c}^{2}}{n \alpha_{c}} c=n \alpha_{c} c
$$

from which it follows directly that

$$
v v_{n}=c^{2}
$$

It follows from the above formulas that operating with the least intervals $l_{i}$ and $\tau_{o i}$, correspondingly, for the transversal motion by the quantity $\lambda$ in this way, one can use the potential $H_{i}$ as the equivalent variables or the number $n$. It follows from the equation (55) that the velocity of transversal motion is greater than the speed of light. 


\section{On the Content of the Concept of "Mass"}

We now proceed to the disclosure of the content of the fundamental property of the matter, the "mass", based on the content of the parameters characterizing the mechanism of motion. Let us represent the equations of motion (29), (31) and (22) in the following form

$$
\frac{\lambda H_{i}}{\pi H_{0 S}^{2}}=\xi_{d}, \quad \frac{(\alpha 2 \pi r+\lambda) H_{i S}^{2}}{\left(H_{0}+H_{i}\right) \pi H_{0 S}^{2}}=\xi_{d}, \quad \frac{\lambda_{0} H_{0 S}}{\pi H_{0}^{2}}=\xi_{d}
$$

Multiplying all the terms of relations above on the velocity of photons $c=\xi_{d} / \xi_{\tau}(38)$, and taking into account the equations (37) and (47), we obtain

$$
\frac{\lambda c H_{i}}{\pi H_{0 S}^{2}}=c \xi_{d}, \frac{(n \alpha+1) \lambda v H_{i}}{\pi H_{0 S}^{2}}=c \xi_{d}, \frac{c \lambda_{0}}{\pi H_{0}^{2}}=c \xi_{d}
$$

where is denoted $H_{0} \xi_{d}=\lambda_{0}$.

Multiplying the nominators of above expressions by the constant quantity $\xi_{m}$ with the dimension of mass, with the allowance of equations $H_{0}+H_{i}=(n \alpha+1) H_{i}, H_{i} \xi_{d} / H_{i} \xi_{\tau}=c, H_{0} \xi_{d} / H_{0} \xi_{\tau}=c$ we obtain

$$
m_{i} c \lambda=h, \quad m v \lambda=h, \quad m_{0} c \lambda_{0}=h
$$

or

$$
c \lambda_{0}=h m_{i}^{-1}, v \lambda=h m^{-1}, v \lambda_{0}=h m_{0}^{-1}
$$

where the mass of interaction $m_{i}$, the total mass $m$ and the rest mass $m_{0}$ are defined by the formulas

$$
m_{i}=\frac{\xi_{m} H_{i}^{2}}{H_{i} \pi H_{0}^{2}}=\frac{\xi_{m} H_{i}}{\pi H_{0}^{2}} ; \quad m_{0}=\frac{\xi_{m} H_{0}^{2}}{H_{0} \pi H_{0}^{2}}=\frac{\xi_{m}}{\pi H_{0}} ; \quad m=\frac{\xi_{m}\left(H_{0}+H_{i}\right)^{2}}{\left(H_{0}+H\right) \pi H_{0}^{2}}=\frac{\xi_{m}\left(H_{0}+H\right)}{\pi H_{0}^{2}}
$$

the constant $h$ is a combination of $\xi_{d}, \xi_{t}$, and $\xi_{m}$ :

$$
h=\frac{\xi_{m} \xi_{d}^{2}}{\xi_{\tau}}
$$

According to the definitions (58) or (59), the mass is a result of unifying of transversal and longitudinal motions characteristics; from definition (60) one is represented by the relations of orthogonal surfaces to the trajectorial volume or, by the corresponding potentials of interaction to the orthogonal surface of the longitudinal motion.

It follows from the equation (60) that the total mass can be represented as the following sum

$$
m=m_{0}+m_{i}
$$

In the absence of the rest mass, it follows from the previous equations (58) and (62) that the particle is moving with the velocity of light. Because the photons rest mass is also absent and characterized by the velocity $c(38)$, one can conclude that the mass of photon is defined by the first formula of the sequence (60). Hence, it follows that the equations of (58) are the equivalent of the De Broglie equations; correspondingly, the numerical value of the Planck constant is defined with the aid of (61).

The final trajectory of $\gamma_{0}$-particles taking part only in their proper interaction (4) is always closed. Thereby, if to observe the particle during $\tau_{0^{-}}$interval or multiple to $\tau_{0}$-intervals, and one fixes the particle in the state of rest, therefore, $m_{0}$ is called the rest mass.

Depending on the nature of revealing, in Physics we operate with the gravitational and inertial masses. The gravitational mass is the quantitative criterion of the force of interaction of physical body with external gravitational fields, and the gravitation field created by the body itself. In the variant set, one considers the different variants of manifestations just the inertial mass.

If we assume that some physical body consists of $N_{m} \gamma_{0 i}$-particles, its mass can be represented by the equation

$$
M=\frac{N_{m}\left(H_{0}+H_{i}\right)}{\pi H_{0}^{2}}
$$


However, such an estimate is purely averaged one; for proper calculation of the mass of particles, there is a need for more information about their structure. Nevertheless, one can always choose some averaged potential $H_{1}$ for simplified calculations (for example, starting from the atomic unit of mass)

$$
M_{1}=\frac{N_{m 1}\left(H_{1}+H_{0}\right)}{\pi H_{0}^{2}},
$$

where $N_{m 1}$ is the number of structural units with the potential $H_{i}=H_{1}$. It follows from the mechanisms of syntheses of elementary particles [9] that the protons are the sources generating the gravitational interaction (the particles of gravitational field), correspondingly, for this case, the gravitational mass $M_{G}$ will be defined as

$$
M_{G}=\frac{N_{m p} H_{p}}{\pi H_{0 S}^{2}}
$$

where $N_{m p}$ is the numbers of protons, $H_{p}$ the number of $\varepsilon$-acts characterizing the integrity of protons. The equality of momenta follows from the first two formulas of the series (58),

$$
m_{i} c=m v
$$

whence it follows that the amplitude of momentum is determined exclusively by the interaction mass.

Differentiating equation (65) by time, we obtain the basic law of mechanics in the conventional form

$$
F=c \frac{d m_{i}}{d t}=\frac{d m v}{d t}
$$

where the force $F$ is determined by the change of interaction mass.

The form and content of equations (65) and (66) do not change at all when passing from the mass $\gamma_{0 i}$-particle to the masses (63) or (64). Just by differentiating the momenta, it is necessary to emphasize that $N_{m}$ (or $N_{m 1}$ ) remain constant, that is, the body under consideration retains its integrity.

From the above, it follows that the multiplying some dimensionless unit by the constant $\xi_{d}, \xi_{\tau}$ (or $\xi_{t}$ ) and $\xi_{m}$ gives this quantity the dimensions of length, time and mass. From here, the constants with the symbol $\xi$ are called the coefficients (or operators) of dimensionalities. The index at $\xi$ indicates the notion used conventionally. It is evident that with the aid of $\xi_{d}, \xi_{\tau}$ and $\xi_{m}$ one can combine the coefficient of any dimensionality.

By composing a coefficient with the dimension of energy

$$
\xi_{\varepsilon}=\frac{\xi_{m} \xi_{d}^{2}}{\xi_{\tau}^{2}}=\xi_{m} c^{2}
$$

and multiplying it by dimensionless components of equation (60), we obtain

$$
\varepsilon_{i}=m_{i} c^{2}, \varepsilon_{0}=m_{0} c^{2}, \varepsilon=m c^{2},
$$

where the energies of corresponding variants of interaction are denoted as

$$
\varepsilon_{i}=\frac{\xi_{\varepsilon} H_{i}}{\pi H_{0}^{2}}, \quad \varepsilon_{0}=\frac{\xi_{\varepsilon}}{\pi H_{0}}, \quad \varepsilon_{m}=\frac{\xi_{\varepsilon}\left(H_{0}+H_{i}\right)}{\pi H_{0}^{2}}
$$

Multiplying $\xi_{\nu}(41)$ by the constant $h(61)$, we obtain the coefficient with the dimension of energy:

$$
\frac{\xi_{m} \xi_{d}^{2}}{\xi_{\tau}} \frac{1}{\xi_{\tau}}=\frac{\xi_{m} \xi_{d}^{2}}{\xi_{\tau}^{2}}=\xi_{m} c^{2}
$$

correspondingly, multiplying all equations (40) by $h$, we will obtain the equalities

$$
h \nu_{i}=m_{i} c^{2}, h \nu_{0}=m_{0} c^{2}, h \nu=m c^{2}
$$

It follows from the equations (68) - (70) that the equivalence of mass, the frequency of manifestation of the integrity and the energy is caused by the identity of their dimensionless components. 


\section{The Numerical Values of the Dimensional Coefficients, $\alpha_{c}, H_{0}$ and $H_{c}$}

In the light of the circumstances that $e^{-}$consists of two $\gamma_{0 i}$-particles $(4)$ in the state of self-interaction, the equation (22) can be represented after elementary transformations as

$$
\frac{1}{\pi H_{e 0}}=\frac{\xi_{d}}{\alpha_{c} 2 \pi r_{e c}}
$$

where we denoted $H_{e 0}=H_{0} / 2, \quad r_{e c}=r_{c} / 2$.

Multiplying both parts of the previous equation by the coefficient of energy $\xi_{\varepsilon}(67)$, we obtain

$$
m_{e} c^{2}=\frac{c h}{\alpha_{c} 2 \pi r_{e c}}=\frac{e^{2}}{r_{e c}}
$$

where, with allowance of (4), (38), (61), (63) and (67), the mass of $e^{-}$is defined by the formula

$$
m_{e}=2 \xi_{m} / \pi H_{0}=\xi_{m} / \pi H_{e 0}
$$

Starting from the notion $\hbar=h / 2 \pi$ and the known relation $c \hbar \alpha=e^{2}$ between the fine structure constant $\alpha$ and the charge from equation (73) one can conclude, that

$$
\alpha_{c}=\alpha^{-1}
$$

that is, the constant defined by expression (8) is the inverse quantity of the fine structure constant $\alpha$.

Using the equations (38), (61), (73) and (74) let us wright

$$
\frac{e^{2}}{G m_{e}^{2}}=\frac{c \hbar H_{e 0}^{2}}{G \alpha_{c} \xi_{m}^{2}}=\frac{\xi_{d}^{2}}{G 2 \pi \xi_{m} \xi_{\tau}^{2}} \frac{H_{e o}^{2}}{\alpha_{c}}
$$

Considering the equation (76) as the ratio of energies of electrostatic and gravitational interactions for the same distances $G$, one can conclude that the Newton's gravitational constant $G$ is defined by the formula

$$
G=\frac{\xi_{d}^{3}}{2 \pi \xi_{m} \xi_{\tau}^{2}}
$$

In this case, the numerical value of $\pi^{2} H_{e 0}^{2} / \alpha_{c}$ is equal to the quantity, which was named by Eddington as the Constant of the Universe.

Using equations (38), (61) and (77), the numerical values of coefficients $\xi_{d}, \xi_{\tau}$ and $\xi_{m}$ can be defined by the formulas

$$
\begin{gathered}
\xi_{d}=\left(\frac{2 \pi G h}{c^{3}}\right)^{1 / 2} \approx 1.015 \cdot 10^{-34} \mathrm{~m}, \\
\xi_{\tau}=\left(\frac{2 \pi G h}{c^{5}}\right)^{1 / 2} \approx 3.38 \cdot 10^{-43} \mathrm{~s}, \\
\xi_{m}=\left(\frac{c h}{G}\right)^{1 / 2} \approx 2.176 \cdot 10^{-8} \mathrm{~kg},
\end{gathered}
$$

whence, it follows that $\xi_{m}=m_{p}, \xi_{d}=2 \pi m_{p}, \xi_{\tau}=2 \pi t_{p}$, where $m_{p}, l_{p}$ and $t_{p}$ are the Planck's units of mass, length and time [11].

Starting from the formulas of calculation of $m_{e}(74)$ and $\xi_{m}(78)$, the numerical values of the constants are the following

$$
H_{e 0}=\left(\frac{c h}{G m_{e}^{2}}\right)^{1 / 2}=2.3887 \cdot 10^{22}, \quad H_{0}=2 H_{e o}=4.777 \cdot 10^{22}, \quad H_{c}=H_{0} / \alpha_{c}=3.46 \cdot 10^{20},
$$

where the value of $\alpha_{c}=137.03699$ [11] has been used. 


\section{Correspondence with Classical Mechanics}

Because of the orthogonality of vector parameters of longitudinal and transversal motion, the results of all products in formulas (58) are the axial vectors. Let a $\gamma_{0 i}$-particle travels the longitudinal path $\lambda$ with the velocity $v$. In this case, the product $m v \lambda=h$ is a scalar with the dimension of action $S$. With respect to the single particle with the momentum $P=m v$, the action $S$ is given by the integral $S=\int_{q_{0}}^{q} P d q$, where $q$ is the transversal path. The real trajectory of the motion relative to the single particle is determined by the condition $\delta S=0$ (the principle of least action), which in turn can be obtained from the following considerations. By the uniform element discretization of the path of particle $q, \lambda=q_{1}-q_{0}=q_{2}-q_{1}=\ldots=q_{n}-q_{n-1}$ and transforming the equation (58) for the general case as

$$
m v \lambda=h
$$

the action $S$ is defined as the sum

$$
S=\int_{q_{0}}^{q_{1}} m v d q+\int_{q_{1}}^{q_{2}} m v d q+\ldots+\int_{q_{n-1}}^{q_{n}} m v d q=n h
$$

and, correspondingly,

$$
\delta S=\delta \int_{q_{0}}^{q_{1}} m v d q+\delta \int_{q_{1}}^{q_{2}} m v d q+\ldots+\delta \int_{q_{n-1}}^{q_{n}} m v d q=n \delta h=0
$$

because $\delta h=0$. Thus, if the trajectory of motion of a particle with the momentum is plotted by the smallest intervals of path $\lambda$ or $n \lambda$, at the any stretch of this trajectory, the particle is identified by the equations (80) and (82). Because the fulfillment of condition (82) is equivalent to the action of laws in the $\mathrm{CM}$, one operates only with the parameters of longitudinal motion; one can conclude that in the frames of the CM just the longitudinal motion is usually investigated. In the CM frequently operate with the notion of the rectilinear motion. It follows from the equations of motion (22), (29) and (31) that those physical bodies do not possess in the least the rectilinear motion, however, at some values of the used intervals of the path and time, the orthogonal surfaces and the transversal motion may become non-observable. According to (82), the least interval of path in the CM is equal to $\lambda$. Correspondingly, for the intervals of longitudinal motion essentially exceeding $\lambda$ both the transversal components of trajectory and the orthogonal surfaces will be non-observable. Numerically, the large values of $n$ in the formula (81) correspond to this condition $S=n h$. The same condition specifies the transition from the CM to the QM and describes the motion by means of the Feynman integrals [12]. Summing up, we can conclude that the physical body is in a state of the rectilinear uniform motion with the constant velocity and the potential unaltered in magnitude and direction.

\section{Correspondence with Quantum Mechanics}

When operating with the spatial and temporal intervals comparable with the proper kinematical characteristics of particles (32)-(34) and (39), the transversal component of motion becomes observable. Hence, the transversal path $\lambda$ of identification particles becomes an important parameter in equation of description of motion already from a comparison of the contents of equations. In the same equations, the parameter $\lambda$ is the length conjugate with the particle of the wave according to the interpretation apparatus of quantum mechanics. Therefore, according to the de Broglie equations of the QM, the equations (58) are the quantitative base of the dual nature of the matter; however, it follows from the method of obtaining these equations that not the Nature of matter is dual but the mechanism of motion of physical particles. Moreover, the formulas (58) or (59) relate the parameters of the longitudinal $v$ and the transversal motion $\lambda$. The results of this relation is the origination of the fundamental property of matter, namely the mass. From the equation (58), the Heisenberg uncertainties principle $\Delta p \Delta l \sim \hbar$ and $\Delta \varepsilon \Delta t \sim \hbar$ can be simply deduced, and the meaning of those is the following: the lesser is the intervals of path and time, which are necessary to identify the integrity of particles, the greater is the rate of uncertainty of revealing their momentum or energy. 
Let us consider several examples frequently discussed in the QM in view of the proposed mechanism of motion. In the QM, the product of velocity of particle motion $v$ and the phase velocity related to the particle of the wave $v_{p h}$ is equal to $v v_{p h}=c^{2}$, from which it follows that the phase velocity $v_{p h}$ has no physical meaning, because it is greater than the speed of light in vacuum $c$. It follows from the equation (55) that $v_{p h}=v_{n}$, which has the clear interpretation: the velocity of transversal motion is greater than the speed of light in vacuum because the orthogonal surface of transversal motion is lesser than the orthogonal surface of the longitudinal motion.

To form the interpretation apparatus of quantum mechanics, the most important role has been played by the explanation interferention or diffraction of particles (including those with the rest mass) on the crystal lattices. Currently, the phenomenon of diffraction is explained by the result of the formulation of quantum mechanics with the use of the path integrals [12], and not by means of the dual nature of matter, despite the idea of the possibility of simultaneous following of physical objects along many trajectories is not tangible in the same way as the dual nature of matter. In explaining the diffraction in the framework of our approach, the geometric and structural aspects of the phenomenon are clearly distinguished. The structural component of diffraction is formally analogous to the Huygens-Fresnel principle; however, the particles and not the waves are incident on the surface of crystal. These particles, interacting with the centers of the media are forming the complexes, the secondary sources of emission of new particles and not the waves. Hence, the resulting diffraction pattern reflects the ordering of organization of mentioned centers of crystal lattice.

The geometric aspects of diffraction are related to the three features:

1. the trajectory of particles of an incident beam is not the rectilinear

2. the surface, which is embraced by a trajectory of transversal motion, is orthogonal to the direction of motion of particles of an incident beam

3. the path length of identification of transversal motion $\lambda$ is compatible with the parameter of diffraction lattice.

Now, based on the listed structural and geometrical peculiarities, let us consider the diffraction of light on the surface of the crystal lattice. In the ST, the mechanism of formation of spatially related systems, including the crystal bodies, is explained by the generalization principle $[9,10]$. Initially, accordin to the scheme analogous to (5), the specific $e^{-}$interacting with the specific $p^{+}$of the nucleus form the complexes $\left(e_{i} / \gamma_{p M}\right)-\left(\mid \gamma_{e M} / p_{i}\right)$ where $e_{i}$ and $p_{i}$ denote the bases of $e^{-}$and $p^{+}$in the state of interaction, $\gamma_{e M}$ and $\gamma_{p M}$ are the derivatives of $\gamma_{e E}$ and $\gamma_{p E}$ particles, with the participation of which the electrostatic interaction is realizing. In the cases, when some complex with two $\gamma_{e M}-\gamma_{p M}$ particles of the type $\left(e_{i} / 2 \gamma_{p M}\right)-\left(2 \gamma_{e M} / p_{i}\right)$ is formed with the participation of the specific $e_{i}-p_{i}$ pair, one of the $\gamma_{e M} / \gamma_{p M}$ pair stands out from the system in the form of photon (the scheme (6)) or transferred as heat to the particles of the surrounding media. The remaining pair becomes common for both $e_{i}-p_{i}$ bases, which become bound. In the case of spatial generalization, the $\gamma_{e M}$ and $\gamma_{p M}$ from given base participate in the generalization with the presence of two and more $e_{i}-p_{i}$ pairs, thereby the $e_{i}-p_{i}$ pairs become bound (that is, the centers of generalization) of all system. The energy of bound created by the generalization is equal to the energy stand off (or remained) $\gamma_{e M} / \gamma_{p M}$-pair. Let $\gamma_{e M}, \gamma_{p M}$-pairs participate during the cycle of integrity in the creation of bounds between $n_{d}$ centers with the values of the transversal path between them equal to $\lambda_{d}$. Then, taking into account the condition of the formation of the transversal component of the closed figure, that is, of the circle (50), one can write

$$
2 \pi r_{d}=n_{d} \lambda_{d}
$$

where $r_{d}$ is the radius of circle. The trajectories of the transversal motion of $\gamma_{e m} / \gamma_{p m}$-particles between the centers bound by the generalization are called the bound lines. On the basis of formula (83), the maximum remoteness $d$ between two centers in the group of $n_{d^{-}}$centers is determined by the following formula

$$
d=\frac{2 \pi r_{d}}{2}=\frac{n_{d} \lambda_{d}}{2} \quad \text { or } \quad 2 d=n_{d} \lambda_{d}
$$

During diffraction, the part of the incident photons in the light beam is reflected from the crystal lattice; the other part, interacting with the centers of the surface decomposes into the compound particles 
$\gamma_{e f}$ and $\gamma_{p f}$ (with the aid of the index " $\mathrm{f}$ " it is denoted that these and $\gamma_{p f}$ particles are originated owing to the photons decay).

The direction of incidence of the beam corresponds to the longitudinal motion, the surface of the closed transversal motion is perpendicular to the beam direction, besides, for the $\gamma_{i} \lambda$-interval (30) the path of transversal displacement $n^{2} \alpha^{2}$ time is greater than the longitudinal paths (32). Hence, after the photon decay on the centers of lattice, its compound particles $\gamma_{e f}$ and $\gamma_{p f}$ are penetrating into the region of geometrical shadow owing to the motion along the bound lines. Further, $\gamma_{e f}$ and $\gamma_{p f}$ particles interacting with the centers of lattice create new complexes, that is, the centers of secondary radiation in the region of geometrical shadow. Because the longitudinal path of penetration deep into the crystal body is essentially lesser than the transversal part, the penetration of $\gamma_{e f}$ and $\gamma_{p f}$ particles into the region of geometrical shadow is realized mainly at the surface of the lattice; hence, the centers of secondary radiation are formed chiefly on the surface of the diffraction gratings. Most likely, this fact can explain the diffraction of light in the $2 \mathrm{D}$ systems of the graphene type.

At the centers of secondary radiation, new photons can be formed by $\gamma_{e f}$ and $\gamma_{p f}$ particles, as the same and the different photons. Because the bound state in photons is caused exclusively by the self-consistent motion of compound $\Delta$ - elements, the newly combinable $\gamma_{e f}$ and $\gamma_{p f}$ particles should have strictly equal energy and phases of motion. This explains more clear diffraction pattern when the monochromatic light is used.

To provide the penetration of $\gamma_{e f}$ and $\gamma_{p f}$ particles into the region of geometrical shadow, it is necessary to have the comparable bound path $\lambda_{d}$ (83) and $\lambda$ of incident photons, besides, the maximum number of enveloped centers of medium $n_{\max }$ we will define from the value of the resultant bound path

$$
n_{\max } \lambda=n_{d} \lambda_{d}
$$

In the region of geometrical shadow, the centers of secondary radiation relative to the incidence point along the opposite sides are located at the distances $\pm \lambda, \pm 2 \lambda, \ldots \pm m \lambda$, thereby the $m=0, \pm 1, \pm 2 \ldots \pm m$ series can be used as s criterion of definition of the order of the diffraction pattern. At $m=0$, the photons of incident beam are reflecting and do not penetrate into the region of geometrical shadow.

For distances $\lambda / 2,3 \lambda / 2 \ldots m \lambda / 2$, the clear layers are not observed at the screens because there is no centers of radiation at these distances.

In the approach outlined here, the wave properties are not assigned to photons and to the photons streams, correspondingly, the patterns observed at the screen are considered because of the interaction of compound particles of photons with the particles of the medium, and not owing to the superposition of waves.

Let the lines, the directions of those coincide with the directions of beams from the secondary sources to the side of screen are extrapolated in the opposite direction. The point of intersection of these lines will be considered as an imaginary source of radiation, the rays of which by equal angles between themselves are falling on the screen through the secondary centers of radiation. Taking into account that the distance $L$ from the imaginary source to the screen is essentially greater than the distance $r$ to the secondary centers, ony can write

$$
\frac{\lambda}{r}=\frac{x_{0}}{L}, \quad \text { or } \quad x_{0}=\frac{\lambda L}{r}
$$

whence it follows that the distance $x_{0}$ between the illuminated bands is increasing with the increase of $\lambda$ and $L$, and decreases with the increase of $r$.

Let us consider the dependences of diffraction peculiarities on the angle of incidence beam relative to the surface of the lattice. It is evident that at the perpendicular incidence of the beam, the photons are interacting with the maximum number of particle of the medium $n_{\max }$. Let the diameter $D$ and $n_{\max }$ are related as

$$
n_{\max }=\chi D
$$

where $\chi$ is the proportionality coefficient.

The dependence of projection $D$ on the incidence angle $\alpha$ is given by the following equality

$$
D \cos \left(\frac{\pi}{2}-\alpha\right)=D \sin \alpha
$$


where it is taken into account that the diameter of the circle caused by the transversal component of the motion is always perpendicular to the direction of beam incidence.

On the basis of equations (87) and (88), the number $n$ of enveloped centers of lattice depending on $\alpha$ will be defined by the formula

$$
n=\chi D \sin \alpha=n_{\max } \sin \alpha,
$$

Multiplying both sides of obtained equation by $\lambda$ and using the formula (84) and (85) we will get the Wulff-Bragg equation

$$
n \lambda=2 d \sin \alpha
$$

So, the base of explanation of interference and diffraction is the allowance of the complexity of the structure of photon and the presence of the transversal component of the motion, owing to which the photons of an incidence beam interacting with the particles of the medium penetrate into the region of geometric shadow and they create the secondary centers of radiation.

The explanation of spectral lines of hydrogen atom belongs to the revolutionary achievments of the QM. According to the scheme (5), the hydrogen atom in the unexcited state is the particle of electrostatically interacting electron $e^{-}$and proton $\left(p^{+}\right)$, and according to which the electron in the state of interaction is characterized by the $\Delta$-content $2 j_{i} 2 \bar{i} 2 \bar{k}$ with the derivatives of $\gamma_{P E}$-particles $2 \bar{j} 2 i 2 \bar{k}$. According to the mechanism considered above, the base of $e^{-}$virtue of its $\Delta$-content is characterized by the longitudinal and transversal components of motion. The longitudinal motion is oscillation, caused by the leading $2 j_{i}$-pairs of interaction with the orthogonal surface $H_{0 S}^{2}$; the proper $2 \bar{i}$ - and $2 \bar{k}$-pairs are leading to the transversal motion with the orthogonal surface $H_{i S}^{2}$. The final trajectory of the motion of an electron in the hydrogen atom, formed by the summing of oscillations along three mutually perpendicular directions with the parametric equation of the type (19) can be considered as the circulation of the oriented surface along the circumference, perceived as the orbit of the electron. The duration of one cycle of interaction related to the manifestation of the integrity of the $e^{-}$is $\tau_{0}=H_{0} \xi_{\tau}$; correspondingly, after each $\tau_{0}$-interval $\gamma_{e E}$ and $\gamma_{p E}$ particles return to their partners, doing, as a result, the shuttle motion along the circle between the bases $e^{-}$and $p^{+}$. As sovereign units, the bases $e^{-}$and $p^{+}$have their own trajectory after interaction in correspondence with their $\Delta$-content. There is no field which results in the motion of $e^{-}$ and $p^{+}$particles towards each other, any changes of the condition of motion of $e^{-}$and $p^{+}$can be caused only by the changes of their $\Delta$-content. Because the mass of $p^{+}$is essentially greater than the mass of $e^{-}$, the values of the longitudinal and transversal motion are essentially lesser than the parameters of $e^{-}$, thereby, the state of $p^{+}$is taken as the "rest" with respect to $e^{-}$.

In the case in question, the motion of $e^{-}$is considered as a motion of an integer unit with the minimal intervals of the path of the longitudinal $H_{j i} \xi_{d}$ and the transversal motion $\lambda$. To describe the motion of an integer particle, the condition (23), the formula (72) and the action $S$ (scalar) are used as criteria.

To apply the analogous approach, one should to start with the formula (58); in this variant, the action becomes an axial vector, which will be denoted by an arrow over the symbol $S$ :

$$
\boldsymbol{S}=\oint_{L} m_{e} v d l=n \boldsymbol{h}
$$

where the Planck constant $h$ also becomes an axial vector, $d l=d l_{i}+d l_{k}(13), v$ is the velocity of the longitudinal motion. The path integration along $L=2 \pi r$ results in the condition of quantization of the angular momentum

$$
m_{e} v r=n \hbar
$$

where $\hbar=h / 2 \pi$.

It follows from the equality $\boldsymbol{S}=n \boldsymbol{h}$ that the circulation path $2 \pi r$ is the sum of $n$-fold repetitions of the path $\lambda$ :

$$
2 \pi r=n \lambda
$$

This formula was obtained earlier (50) from the condition of describing the motion of integer particles. 
Let us also define the following characteristics of $e^{-}$using the equations (44), (48), (53) and (68)

$$
\begin{array}{r}
\lambda=n \alpha_{c}^{2} H_{e c} \xi_{d}, \quad 2 \pi r_{n}=n^{2} \alpha^{2} H_{e c} \xi_{d}, \quad v_{n}=\frac{c}{n \alpha_{c}+1} \approx \frac{c}{n \alpha_{c}} \\
\varepsilon_{n}=\frac{m_{e} c^{2}}{2 n \alpha_{c}\left(n \alpha_{c}+1\right)} \approx \frac{m_{e} c^{2}}{2 n^{2} \alpha_{c}^{2}}, \quad n \alpha_{c} \gg 1
\end{array}
$$

where the quantized parameters are indexed by $n$, with regard to the $\gamma$-content of $e^{-}$, its mass is defined by the formula (74); it is assumed that $H_{e c}=H_{e} \xi_{d} / 2$.

It is not difficult to see that the set of equations (92), (93) and (94) coincides with the wave-mechanical equations of $e^{-}$description on the stationary Bohr orbits. Hence, the most important conclusion follows: the Bohr orbits are the manifestation of the transversal component of the motion. Hence, the answer follows on the question: why $e^{-}$does not fall on the $p^{+}$because of the electrostatic attraction? Simply, because of its $\Delta$-content, the trajectories of $e^{-}$and $p^{+}$are such that this event is simply excluded. The nature of the Coulomb attraction and repulsion manifests itself as applied to the physical bodies with the essentially big charges $[9,10]$.

Because the orbit of $e^{-}$in the $\mathrm{H}$ atom is represented as a trajectory of transverse motion, the quantum numbers characterizing the orbits should obtain the corresponding content, too. In the case of absorption of photons by the hydrogen atom, the new partners arise and the process of $\Delta$-pairs exchange with the $e^{-}$ takes place. Thereby, the orbit of $e^{-}$is formed not only by the proper $2 i$-and $2 k$ pairs but the extraneous $\Delta_{k f}$ - and $\Delta_{j}$-pairs (the symbol $f$ near the index of $\Delta$ points out the extraneous origin of the $\Delta_{k}$-pair). As a result, the transversal path of identification $\lambda$ is determined by the sum of portions introduced by the all $\Delta$-pairs:

$$
\lambda=\lambda_{i}+\lambda_{k}+\lambda_{k f}+\lambda_{j},
$$

and the length of the closed transversal path is represented as

$$
L_{n}=n \lambda=n\left(\lambda_{i}+\lambda_{k}+\lambda_{k f}+\lambda_{j}\right)
$$

or, taking into account the formulas $(94),(95)$ and (96),

$$
L_{n}=\frac{1}{2} n\left(n_{i}+n_{k}+n_{k f}+n_{j}\right) \cdot \alpha_{c}^{2} H_{c} \xi_{d}
$$

where is denoted

$$
\begin{gathered}
\lambda_{k}=n_{i}+n_{k}+n_{k f}+n_{j} \\
\lambda_{i}=\frac{1}{2} n_{i} \alpha_{c}^{2} H_{c}, \quad \lambda_{k}=\frac{1}{2} n_{i} \alpha_{c}^{2} H_{c} \xi_{d}, \quad \lambda_{k f}=\frac{1}{2} n_{k f} \alpha_{c}^{2} H_{c} \xi_{d}, \quad \lambda_{j}=\frac{1}{2} n_{j} \alpha_{c}^{2} H_{c} \xi_{d} .
\end{gathered}
$$

Because the paths $\lambda_{i}$ and $\lambda_{k}$ are formed by the proper $2 i$ - and $2 k$-pairs, $\lambda_{k f}$ and $\lambda_{j}$ by exterior pairs, $n_{i}$ and $n_{k}$ are called the proper and $n_{k f}$ and $n_{j}$ the exterior quantum numbers and because the moduli of proper initial potentials $H_{0 i}$ and $H_{0 k}$ are equal, the following condition is always met

$$
n_{i}=n_{k},
$$

besides, for any orbit there are the presence of minimal values $n_{i}=n_{k}=0.5$, or

$$
n_{i}+n_{k}=1
$$

is mandatory, because the fulfillment of this condition is the criterion of identity of an investigated electron in the atom of hydrogen.

It follows from the notations (98), (100) and (101) that the sum of exterior quantum numbers

$$
\ell=n_{k f}+n_{j}
$$

obtains the integer values from zero to $n-1$ and indicates the number of exterior partners with the participation of which the length of transversal path is formed. Denoting

$$
m_{\ell}=n_{k f}-n_{j}
$$


one can make sure that $l$ corresponds to $2 \ell+1$ variants of realization of the predetermined number, because $m_{e}$ obtains the following values: $m_{e}=0, \pm 1, \pm 2, \ldots \pm, \ell$.

It is evident that the parameters $\ell$ and $m_{\ell}$ defined by the formulas (102) and (103) correspond to the azimuthal and magnetic quantum numbers.

The quantum numbers $n_{i}, n_{k}, n_{k f}$ and $n_{j}$ are related to the interaction potentials along three mutually orthogonal directions, that is, with the potentials of $2 i-, 2 k-, 2 k_{f}-$ and $2 j-$ pairs and taking into account the equality (96) one can not only to replace the quantum numbers $n, \ell$ and $m_{\ell}$ but to give them a new additional content. From (98), (102) and (103), the group with the quantum number $n$ can be realized by

$$
\sum_{\ell=0}^{n-1}(2 \ell+1)=n^{2}
$$

variants, and the total number of ways to fill all the groups

$$
\sum_{n=1}^{n} n^{2}=\sum_{n=1}^{n} \sum_{\ell=0}^{n-1}(2 \ell+1)
$$

With the help of sum (105) we determine the number of variants in the group with the given principal quantum number $n$. Each $\Delta$ - content, which is realized by the interaction with the concrete partner, corresponds to each variant. Hence, with the use of quantum numbers $n_{k f}$ and $n_{j}$ we determine, the number of partners and what $\Delta$ - content forms the state under consideration. This is especially important when modelling the nuclei and multielectron atoms. Summing (105) in limits $n=1-7$, we obtain the number 140 , which is the key parameter of modelling of $\Delta$ - elements and $\Delta$-pairs from $\varepsilon$-particles; the same number, according to notations (2) and (8) are the base to obtain the fine structure constant. From the foregoing follows that the quantum numbers became the universal regulators of structure formations for the systems of various hierarchical levels; besides, the given interpretation has a generality and can be applied to any system of macro- and microworld.

We now turn to the derivation of the basic equations of the QM, based on the proposed mechanism for the motion of the $\gamma_{0 i}$ - particles. Investigating physical phenomena, the durations of periodically repeating processes frequently are used as an etalons relative to which the different temporal changes are considered. Because the transversal component of motion is characterized by the periodically repeating closed curvilinear trajectory, the duration of one cycle may be fully considered as an etalon when describing the regularities of motion, especially, if the objects under consideration are being considered as a reference frame.

Let the potentials of $\gamma_{0 i}$-particles caused by the $2 i$ - and $2 k$-pairs are characterized by equations

$$
\begin{aligned}
& H_{i i}=H_{i 0} \cos \omega\left(t+t_{0}\right), \\
& H_{k i}=H_{k 0} \sin \omega\left(t+t_{0}\right)
\end{aligned}
$$

where $\omega=2 \pi / \tau_{0 i}, \tau_{0 i}$ is the period of revealing of the integrity, $t$ is time, $t_{0}$ is an initial time. If the path traveled by time $t_{0}$ with the velocity $v=\lambda / \tau_{0 i}$ is equal to $x$, then $t_{0}=x \tau_{0 i} / \lambda$ and the equations (106) will be represented in the form

$$
\begin{aligned}
& H_{i i}=H_{i 0} \cos (\omega t+k x), \\
& H_{k i}=H_{k 0} \sin (\omega t+k x)
\end{aligned}
$$

where $k=2 \pi / \lambda$.

Multiplying and dividing $\omega$ and $k$ by the constant $\hbar$ and taking into account (33), (61), (67) and (71), we obtain

$$
\begin{aligned}
& H_{i i}=H_{i 0} \cos \frac{i}{\hbar}\left(\varepsilon t+P_{x} x\right), \\
& H_{k i}=H_{k 0} \sin \frac{i}{\hbar}\left(\varepsilon t+P_{x} x\right)
\end{aligned}
$$

where $P_{x}$ is the projection of momentum along the $x$ direction. 
With allowance of the condition $H_{i i} \perp H_{k i}$, we can write for the total potential $H_{i}^{2}$

$$
H_{i}^{2}=H_{i i}^{2}+H_{k i}^{2}=H_{i i}^{2}-\left(i H_{k i}\right)^{2}=\left(H_{i i}+i H_{k i}\right)\left(H_{i i}-i H_{k i}\right)
$$

from which it follows with the allowance of equations (108) and the Euler formula,

$$
H_{i}^{2}=H_{0}^{2} \exp \left(-\frac{i}{\hbar}\left(\varepsilon t-P_{x} x\right)\right) \exp \left(\frac{i}{\hbar}\left(\varepsilon t-P_{x} x\right)\right)=\psi \psi^{*}
$$

where we denoted

$$
\psi=H_{0} \exp \left(-\frac{i}{\hbar}\left(\varepsilon t-P_{x} x\right)\right), \psi^{*}=H_{0} \exp \left(\frac{i}{\hbar}\left(\varepsilon t-P_{x} x\right)\right)
$$

Twice differentiating $\psi$ by coordinate $x$, we obtain

$$
\frac{\partial^{2} \psi}{\partial x^{2}}-\frac{p^{2} \psi}{\hbar^{2}}=0
$$

whence, with allowance of definition of the Laplace operator $\nabla^{2}=\partial^{2} / \partial x^{2}+\partial^{2} / \partial y^{2}+\partial^{2} / \partial z^{2}$ we obtain the stationary Schrödinger equation

$$
\nabla^{2} \psi-\frac{8 \pi^{2} m}{h^{2}}(E-U) \psi=0
$$

where $E$ and $U$ are the total and potential energies, $E-U=p^{2} / 2 m$.

The transition to the operators of momentum $\hat{P}_{x}$ and energy $\hat{\varepsilon}$ is made with the aid of partial derivatives of $\psi$ by coordinate and time.

$$
\hat{P}_{x}=-i \hbar \partial / \partial x, \quad \hat{\varepsilon}=i \hbar \partial / \partial t
$$

where the "cap" over the symbol denotes the operator.

It is obvious that the commutativity of the Poisson brackets $\left[x, \hat{P}_{x}\right]$ and $[t, \hat{\varepsilon}]$ will be defined by the ratio of the used least intervals to the proper kinematic parameters.

With the use of operators (114), the average values of momentum $\hat{P}_{x}$ and energy $\hat{\varepsilon}$ are defined by the integrals

$$
\left\langle P_{x}\right\rangle=\int_{V} \psi^{*} \hat{P}_{x} \psi d v, \quad\langle\varepsilon\rangle=\int_{V} \psi^{*} \hat{\varepsilon} \psi d v
$$

Using the normalizing condition,

$$
\int_{V} \psi^{*} \psi d v=1
$$

changing the sequence of factors in the vector product and operating with the moduli of vector quantities, the equation (15) can be represented in the form

$$
\frac{\int_{L}\left(H_{i i}^{2}+H_{k i}^{2}\right) d l}{\pi H_{0}^{3}}=\frac{\int_{L} \psi \psi^{*} d l}{\pi H_{0}^{3}}=1
$$

where the equations (13), (25), (26), (111), and (116) are taken into account.

With allowance of the definitions (58) and (59) the following relations $m v \lambda / h=1$ and $m_{0} c \lambda_{0} / h=1$ also following, which are the criteria of revealing the integrity of particle under consideration, respectively, and the meaning of normalizing results in the statement of revealing of the integrity. In this case, the integration by normalizing should also be carried within the limits of the volume $V_{t}$.

By proper choice of the least intervals of path and time out of the many particles interacting between themselves, the temporal distribution of particles separately taken by an energy can be obtained with the help of the function $\psi$ in the stationary system. Let us consider the possibility of particles penetration through some energy barrier with the value of $\varepsilon_{a}$ greater than the average energy of particles $\langle\varepsilon\rangle$. Let the energies $\varepsilon_{\tau}>\varepsilon_{a}$ exist in the temporal spectrum of particles. The greater the number of intervals $\tau_{0 i}$ 
with the energy $\varepsilon_{\tau}>\varepsilon_{a}$, the greater is the probability of penetration through the barrier with the energy $\varepsilon_{a}$, that is, the periodical function $\psi$ can also be used to solve the problems related to the definition of possibilities of implementation of given events.

Proceeding from the above, one can conclude that the wave function accepted in the QM and the wave function written in (111) are equivalent quantitatively but differ in the content of interpretation. According to the variant set forth, the changes of parameters of longitudinal motion relative to characteristic of transverse motion are considered with the aid of periodic function $\psi$. The phenomena, which within the frameworks of the QM are explained by the presence of the waves conjugate to particles or by an idea of possibility to follow by many trajectories simultaneously for one particle, in the proposed variant is related to the revealing of the transverse motion.

\section{Correspondence with STR}

Particles modeled in the ST are launched into the absolute emptiness; any interactions between particles are realized due to the features inherent in particle models. Hence, any changes in the system under consideration become observable to the researcher. When describing the simulated particles, the bodies of reference are bind to the particles themselves, thereby making it possible to describe the regularities of motion of an individual particle. However, one can consider the motion of a given particle relative to other particles. We will show that when considering the motion of particles with high velocities relative to the motion of particles with lower velocities, we turn from the ST equation to the STR equations.

Up to now, the regularities of motion of separately taken particle have been considered using the mutually orthogonal direction of oscillations $2 i-, 2 j-$ and $2 k$-pairs as the coordinate axes; we determine time as the number of sequentially realized $\varepsilon$-acts along the all direction of motion. Let us consider now the motion of several particles relative to each other. Let all the particles move uniformly and rectilinearly with the constant velocity along the given direction. These conditions are ensured by an introducing the pairs of the same type, coordinated in phase, by selecting the large values of $n$ in $S=n h$ (72). Let us assign to the particles under consideration the axes $x, y, z$, coinciding with the directions of oscillations and consider that the direction of motion coincides with the axe $x$, the coordinates $y$ and $z$ being the same and constant.

Considering the motion of integer particles with different velocities, the distance traveled and the duration of motion, we denote as

$$
\begin{gathered}
x_{1}=n H_{i 1} \xi_{d}, \quad x_{2}=n H_{i 2} \xi_{d}, \ldots x_{p}=n H_{i p} \xi_{d} ; \\
t_{1}=n\left(H_{0}+H_{i 1}\right) \xi_{\tau} ; \quad t_{2}=n\left(H_{0}+H_{i 2}\right) \xi_{\tau}, \ldots t_{p}=n\left(H_{0}+H_{i p}\right) \xi_{\tau}
\end{gathered}
$$

where $p$ is the number of particles. First, consider the motion of two particles. With allowance of (37), we determine their velocities by equations

$$
v_{1}=\frac{x_{1}}{t_{1}}=\frac{n H_{i 1} \xi_{d}}{n\left(H_{0}+H_{i 1}\right) \xi_{\tau}}=\frac{H_{i 1}}{H_{0}+H_{i 1}} c, \quad v_{2}=\frac{x_{2}}{t_{2}}=\frac{n H_{i 2} \xi_{d}}{n\left(H_{0}+H_{i 2}\right) \xi_{\tau}}=\frac{H_{i 2}}{H_{0}+H_{i 2}} c
$$

where $c$ is the velocity of light in free space (38).

Due to the fact that $H_{i} \perp H_{0}$ for particles under consideration, we can write

$$
H_{i 1}^{2}+H_{0}^{2}=\left(H_{0}+H_{i 1}\right)^{2} ; \quad H_{i 2}^{2}+H_{0}^{2}=\left(H_{0}+H_{i 2}\right)^{2}
$$

Multiplying all the terms of the previous equation by the coefficient of dimensionality $\xi_{d}^{2} \xi_{\tau}^{2} / \xi_{\tau}^{2}=c^{2} \xi_{\tau}^{2}$ and further, multiplying and dividing $H_{i 1}^{2}$ by $\left(H_{0}+H_{i 1}\right)^{2}$ and $H_{i 2}^{2}$ by $\left(H_{0}+H_{i 2}\right)^{2}$, we obtain:

$$
\begin{aligned}
& v_{1}^{2} t_{1}^{2}+c^{2} t_{0}^{2}=c^{2} t_{1}^{2}, \quad v_{2}^{2} t_{2}^{2}+c^{2} t_{0}^{2}=c^{2} t_{2}^{2}, \\
& t_{1}=\frac{t_{0}}{\sqrt{1-\beta_{1}^{2}}}, \quad t_{2}=\frac{t_{0}}{\sqrt{1-\beta_{2}^{2}}} \quad \text { or } \quad t_{2}=t_{1} \frac{\sqrt{1-\beta_{1}^{2}}}{\sqrt{1-\beta_{2}^{2}}}
\end{aligned}
$$

where is denoted: $\beta_{1}=v_{1} / c, \quad \beta_{2}=v_{2} / c$. 
Replacing $t_{1}$ and $t_{2}$ in (118) and using (120), we establish the relation

$$
x_{2} \sqrt{1-\beta_{2}^{2}}=\left(x_{1}-v t_{1}\right) \sqrt{1-\beta_{1}^{2}}
$$

When

$$
v_{2} \gg v_{1}, v_{1} \ll c, v_{2}^{2} \approx v^{2},
$$

we obtain the Lorentz transformation from the equation (121) for coordinates

$$
x_{2}=\left(x_{1}-v t_{1}\right) / \sqrt{1-\beta^{2}} .
$$

where $\beta=v / c, v$ is the velocity of body 2 with respect of body 1 .

Denoting $v_{1} t_{1}=x_{1}$ and $v_{2} t_{2}=x_{2}$, we obtain from the equations (120)

$$
x_{1}^{2}-c^{2} t_{1}^{2}=x_{2}^{2}-c^{2} t_{2}^{2}
$$

In the preceding equation, replacing $x_{2}$ by its value in (123) and after elementary transformation, we obtain

$$
\left(\frac{t_{1}-v x_{1}}{c^{2}}\right) \sqrt{1-\beta_{1}^{2}}=t_{2} \sqrt{1-\beta_{2}^{2}}
$$

From the same equation, taking into account (122), we obtain the Lorentz time transformation:

$$
t_{2}=\frac{t_{1}-v x_{1} / c^{2}}{\sqrt{1-\beta^{2}}}
$$

Let us transform the numerator in equation (126) in the form $t_{1}-\frac{v x_{1}}{c^{2}}=t_{1}\left(1-\frac{v v_{1}}{c^{2}}\right) \simeq t_{1}$, where $\frac{v v_{1}}{c^{2}} \ll 1$ is taken into account. From here, we obtain $t_{2}=\frac{t_{1}}{\sqrt{1-\frac{v^{2}}{c^{2}}}}$ which follows also from the equation (120) with the allowance of conditions (122).

Using the definitions of velocity (37), mass (60), and the equality $m v=m_{i} c(65)$ and equation (119), we obtain

$$
m_{1}^{2} c^{2}-m_{1}^{2} v_{1}^{2}=m_{2}^{2} c^{2}-m_{2}^{2} v_{2}^{2},
$$

and

$$
m_{1} \sqrt{1-\beta_{1}^{2}}=m_{2} \sqrt{1-\beta_{2}^{2}}
$$

from which, by applying the condition (122), we obtain the mass transformation, which is conventional in the STR:

$$
m_{2}=m_{1} / \sqrt{1-\beta^{2}}
$$

Using formulas (62) and (65), the squares of momentum and energy follow from the equations

$$
\begin{gathered}
p^{2}=m_{0}^{2} c^{2}+m_{i}^{2} c^{2}=m_{0}^{2} c^{2}+m^{2} v^{2}=m^{2} c^{2}, \\
E^{2}=m_{0}^{2} c^{4}+m_{i}^{2} c^{4}=m_{0}^{2} c^{4}+m^{2} c^{2} v^{2}
\end{gathered}
$$

where the condition $m_{i} c \perp m_{0} c$ (or $m v \perp m_{0} c$ ) is taken into account.

Writing the equation (127) in the following form

$$
m_{1}^{2} c^{2}-p_{1}^{2}=m_{2}^{2} c^{2}-p_{2}^{2}
$$

and using the mass transformations (128), we obtain

$$
p_{2}^{2}=m_{1}^{2} c^{2} \frac{1-\beta_{1}^{2}}{1-\beta_{2}^{2}}-m_{1}^{2} c^{2}+p_{1}^{2}
$$

from here, taking into account the conditions (122), we obtain

$$
p_{2}^{2}=p_{1}^{2}-\frac{m_{1}^{2} c^{2}}{1-\beta^{2}}+m_{1}^{2} c^{2}=p_{1}^{2}+m_{1}^{2} c^{2}\left(\frac{1}{1-\beta^{2}}-1\right)
$$


In this way, taking into account condition (122) and transformations (121), (125), (128) and (133), we turn to the transformations conventional in the SRT. The conditions (122) are met at $H_{i 2} \gg H_{i 1}, H_{i 1} \ll H_{0}$. Further, taking into account the small values of velocity, we turn to the Galilei Relativity $x_{1}=x_{2}, y_{1}=$ $y_{2}, z_{1}=z_{2}, t_{1}=t_{2}$ for the case $H_{i 2} \ll H_{0}$.

We note additionally, that the formulas derived in the intrinsic frame of reference (IFR) are always will be invariant in any inertial reference systems (IRS), because the equations introduced in the IFR are the reflections of structural relationship and changes, independent of such an action as the choice of the IRS, not influencing the process of phenomena under consideration.

\section{Conclusion}

It is assumed that starting from definite hierarchical level, the physical bodies possess the same structural element stipulating the same mechanism and the same quantitative apparatus of describing the motion. The concluding mechanism of motion is represented by the longitudinal and transverse components with the periodically repeating closed curvilinear trajectory. The longitudinal and transverse motions are accompanied by the "making a net" of the corresponding orthogonal surfaces with which the genesis of the fundamental property of matter, the "mass", is related.

The final trajectories of motion are formed by the 3-dimensional figures (for example, by a torus) the volume of which is computed with the help of the basic equation of motion, is an analogue with the Stokes equation by the form but with the cardinally different interpretation. In the approach outlined, the structure of the particle under consideration is used as a reference frame, in connection with which the final equation of motion is always invariant, that is, independent of the choice of the exterior reference frames, which in no way influence the structure of investigated objects.

The passage to the equations of the $\mathrm{CM}$ is defined by the operation of the space-time intervals essentially above the proper kinematical characteristics of physical bodies, in which connection the "made nets" of orthogonal surfaces of longitudinal and transversal motion, like the transversal motion itself, will be unobservable. The analogous space-time intervals operate in the STR, too. While studying the motion at high speeds, when the potentials of external fields of interaction became the quantities of the same order with the potential of the proper interaction, the orthogonality of the direction of displacement caused by the proper and external interactions is revealed. Taken together of moving bodies, assuming the velocity of one of the objects essentially lesser than the speed of light in the vacuum and the velocities of other objects, we obtain the STR transformations.

The passage to the quantitative apparatus of the QM is put into effect by the selection of space-time intervals compatible with the proper kinematical characteristics of physical bodies, thus the transversal component of the motion becomes observable. Hence, the phenomena, which are explained by the presence of the waves conjugate with the particles, are being caused by the transversal components of motion.

\section{References}

1. R. Clifton, Perspectives on Quantum Reality: Non-Relativistic, Relativistic, and Field-Theoretic. SpringerScience-Business Media, ISBN 90-481-4643-7, 2011.

2. M. Dyson, Freeman John ; Moravcsik, Advanced Quantum Mechanics. World Scientific, ISBN 981-4383-40-6, 2011.

3. S. Gielen, D. Oriti, and L. Sindoni, "Cosmology from group field theory formalism for quantum gravity," Phys. Rev. Lett., vol. 111, p. 031301, Jul 2013. [Online]. Available: https://link.aps.org/doi/10.1103/PhysRevLett.111.031301

4. R. Muller, "Why are quantum mechanics and general relativity incompatible?" 2016. [Online]. Available: https://www.quora.com/Why-are-Quantum-Mechanics-and-General-Relativity-incompatible

5. M. Jammer, Concepts of Mass in Classical and Modern Physics. Courier Corporation, 1961.

6. H. Fritzsch, "The problem of mass and mass generation," arXiv preprint hep-ph/9605300, 1996.

7. F. Wilczek, "The origin of mass," Modern Physics Letters A, vol. 21, no. 09, pp. 701-712, 2006.

8. K. Gordon, The Mysteries of MASS. Scientific American, 2005.

9. K. Kirakosyan, Structural Physics. Structural Content of the Basic Concepts and Physical Law. Flinta, ISBN 978-5-9765.-1087-6, 2011. 
10. Kirakosyan, "On the structural content of fundamental properties of matter and universal constants of physics," Theoretical Physics, vol. 3, no. 01, pp. 1-27, 2018. [Online]. Available: https://dx.doi.org/10.22606/tp.2018.31001

11. "The nist reference on constants, units, and uncertainty. codata internationally recommended, 2014 values of the fundamental physical constants." 2016. [Online]. Available: http://physics.nist.gov/cuu/Constants/2016.

12. C. Grosche and F. Steiner, "Handbook of Feynman Path Integrals," Springer Tracts Mod. Phys., vol. 145, pp. 1-449, 1998. 